\title{
Anuria as a Chief Presentation of Takayasu Arteritis, a Case Report
}

\begin{abstract}
Sara KAlrasheed ${ }^{1 *}$, Basel Alheijani ${ }^{1}$, Rahmah Alzahrani $^{1}$ and Shaker Alshehri ${ }^{2}$

${ }^{1}$ Department of Medicine, King Abdulaziz Medical City, Ministry of National Guard-Health Affairs, Saudi Arabia 2Department of Radiology, King Abdulaziz Medical City, Ministry of National Guard-Health Affairs, Saudi Arabia

*Corresponding author: Sara K Alrasheed, Department of Medicine, King Abdulaziz Medical City, Ministry of National Guard-Health Affairs, Saudi Arabia
\end{abstract}

Received: December 10, 2020; Accepted: J anuary 04, 2021; Published: January 11, 2021

\begin{abstract}
Takayasu vasculitis is a rare type of large vessel vasculitis that primarily affects the aorta and its main branches; signs and symptoms are usually due to systemic inflammation or ischemia of an organ or limb, depending on the group of blood vessels involved. In addition, Takayasu arteritis is associated with increased platelet and coagulation activity, leading to a hypercoagulable state and thrombus formation. We report a case of a 47-year-old male who presented with a history of complete anuria for 3 days and was found to have progressively worsening kidney function. Renal Doppler ultrasound confirmed the presence of bilateral renal artery thrombosis, while Contrast- Enhanced Computed Tomography (CECT) of the abdomen and pelvis showed extensive abdominal aortic thrombosis with radiological findings consistent with large vessel vacuities. After catheter-directed thrombolytic therapy of the renal arteries, the patient started producing urine and his kidney function significantly improved. Later, Positron Emission Tomography scan (PET) confirmed large vessel Takayasu arteritis. Echocardiography showed no intracardiac thrombus, along with an extensive work up for thrombophilia, as autoimmune and vasculitis serology came back negative. This is an extremely rare presentation of Takayasu arteritis, with an unusual recovery of acute renal failure after delayed anuria due to bilateral renal artery thrombosis.
\end{abstract}

\section{Background}

Bilateral renal artery thrombosis, secondary to aortic thrombosis is infrequent, often associated with atherosclerotic and/or aneurysmal changes, rarely caused by other conditions. Takayasu large vessel vasculitis (nonspecific) is one of the rare causes of aortic thrombosis [1], and is often missed, although it is a reversible and treatable condition.

\section{Case Presentation}

A 47-year-old male presented with an abrupt onset of anuria for 3 days. There was no history of flank pain, dysuria, hematuria, fever, vomiting, or diarrhea. Ten days prior to his presentation, he was complaining of abdominal pain, mainly around the umbilicus, associated with bloating and constipation. He went to another hospital and was managed with: bisacodyl, oral lactulose, and fleet enema, and was discharged. Review of other systems revealed a history of general fatigue and lower limb claudication after a walking distance of 20 to 30 meters. There was no history of skin rashes, joint pain or swelling, cough, chest pain, palpitation, or shortness of breath. Moreover, there was no history of headache, or any neurological symptoms. His past medical history was significant for hypertension and lumbar spine disk prolapse.

Past medical history was significant for a hospitalization 5 years ago, for unprovoked massive bilateral pulmonary embolism. Investigations at that admission included: thrombophilia workup, which was non-revealing, an echocardiography which showed reduced left ventricular Ejection Fraction (EF) of 45\%. Follow-up Computerized Tomography (CT) of the coronary arteries revealed a zero calcium score and a mild non obstructive Coronary Artery
Disease (CAD) in the proximal Left Anterior Descending Coronary Artery (LAD). Cardiac MRI showed an old infarction in the apical segment, with no Left Ventricular (LV) cavity thrombus. At the time, there was no history of thrombosis (before that presentation), abdominal aortic aneurysm, or renal stones in the patient's history or family. His medications included aspirin, valsartan, metoprolol, and atorvastatin. He did not smoke, use alcohol, or illicit drugs.

Physical exam at presentation: Patient was conscious, oriented, not in pain or distress, body temperature of $36.3^{\circ} \mathrm{C}$, blood pressure: left arm 127/80, right arm 121/78, left leg 57/30, right leg 62/31, heart rate of 79 beats per minute, regular with absent left radial pulse, lower limb pulse was palpable but weak bilaterally, respiratory rate of 21 breaths per minute and pulsoximetry of $98 \%$ on room air. Cardiovascular examinations revealed normal heart sounds, no murmurs, normal JV, no carotid bruit, and no pedal edema. His abdominal exam revealed mild tenderness to palpation, more on the right flank compared to the left: no rebound, guarding, distention, or organomegaly. The rest of the exam was normal.

\section{Investigations}

The initial emergency department evaluation included an Electrocardiogram (EKG), which was unremarkable, a White Blood Cell (WBC) of $7.10 \mathrm{k} / \mathrm{uL}$ (normal range 4-11), a hemoglobin of $136 \mathrm{~g} / \mathrm{dL}$ (normal range 135-180 gm/L), a platelet count $302 \mathrm{~K} / \mathrm{uL}$ (normal range 150-400). Other laboratory investigations revealed a creatinine of $500 \mathrm{umol} / \mathrm{L}$ (normal range 64-110 umol/L), with his last documented baseline was $70 \mathrm{umol} / \mathrm{L}$, a Blood Urea Nitrogen (BUN) of 8 (normal range 7.4-3.2 mmol/L), a potassium of 3.4 (normal range 3.5-5.1 mmol/L), a bicarbonate level of 19 (normal range 22-29 


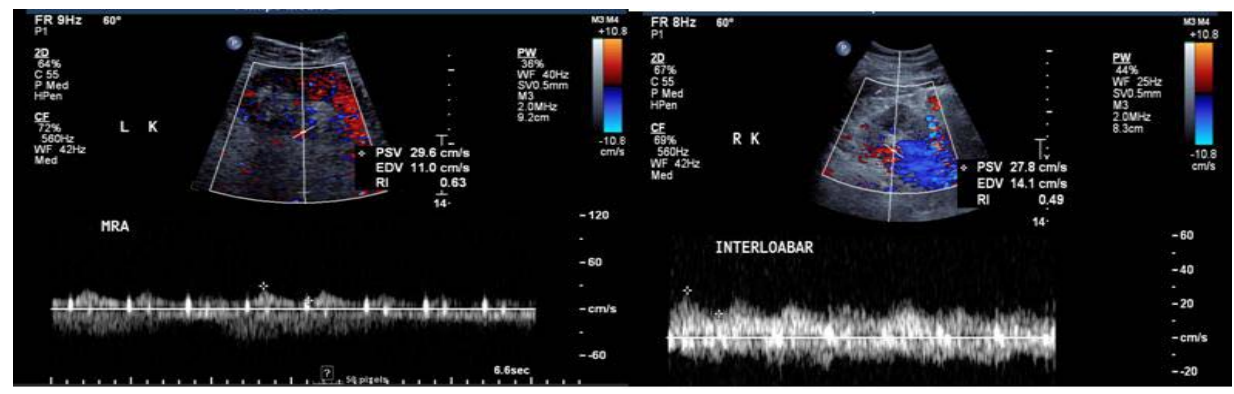

Figure 1: a- Sagittal color Doppler ultrasound of the right kidney (a) interlobar segment shows significant drop in the peak velocity and tardus parvus wave from in addition to low resistive index. (b) Left kidney color Doppler of the main renal artery showed low peak velocity and dampening.

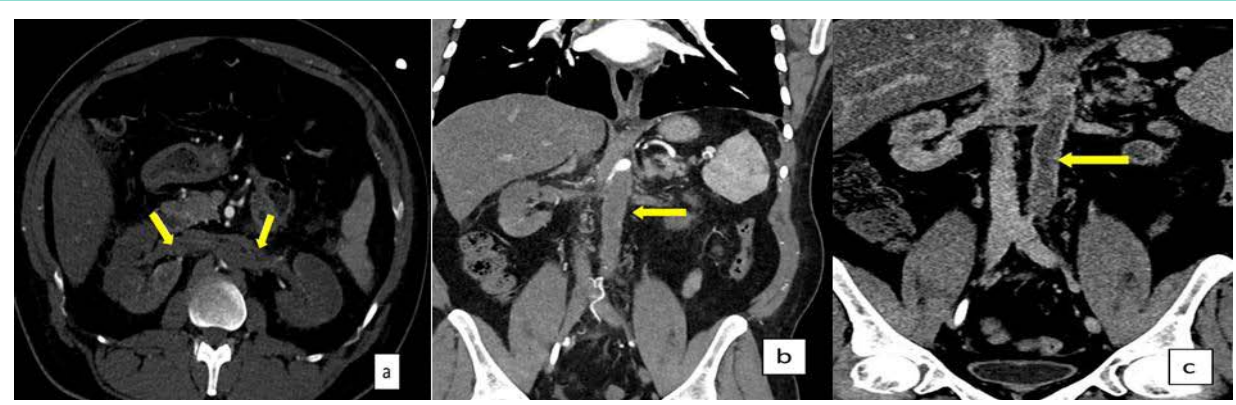

Figure 2: Axial (a) CECT image shows thrombosis of the renal arteries bilaterally (arrow), and coronal (b) image shows infra renal abdominal aorta (arrow) thrombosis extending to the iliac branches. (C) Selected coronal CT shows mural thickening of the infra-renal abdominal aorta and renal artery with delayed enhancement of the wall.

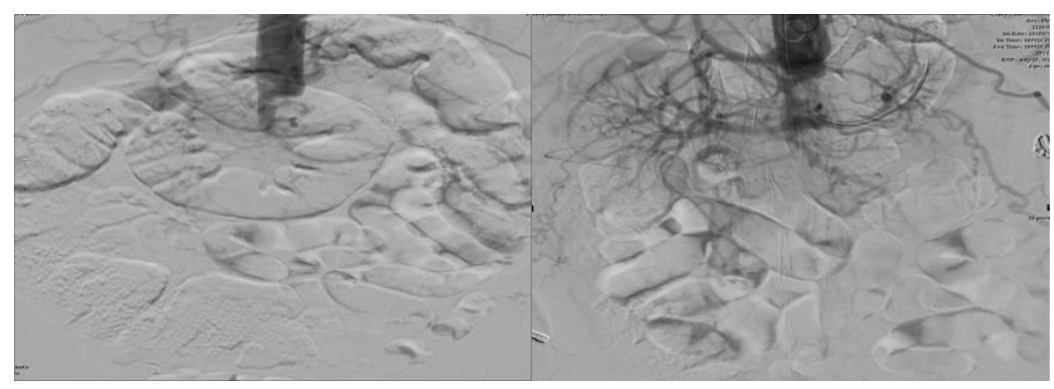

Figure 3: Selective DSA image for the abdominal aortogram from the brachial artery access show occlusion of the infrarenal aorta, renal arteries and revealed total aortoiliac occlusion, bilateral renal arteries and origin of the superior mesenteric arteries thrombosis.

mmol/L). Furthermore, his Lactate Dehydrogenase (LDH) level was 840 (normal range 125-220), and Erythrocyte Sedimentation Rate (ESR) was $49 \mathrm{~mm} / \mathrm{H}$ (normal range $0-15 \mathrm{~mm} / \mathrm{hr}$ ). Other laboratory work-up included liver profile, lactic acid level, and coagulation profile which were within normal limits. Patient was not able to produce urine even after Foley's catheter insertion. Non-enhanced CT of the abdomen and pelvis showed no overt obstructive uropathy or renal stones. On admission, his creatinine level increased to 900 umol/L. Renal Doppler ultrasound was done and showed findings suggestive of evolving bilateral main renal artery thrombosis versus stenosis (Figure 1).

Further assessment by Contrast-Enhanced CT (CECT) of the abdomen and pelvis revealed thrombosis of the renal arteries, the infra-renal abdominal aorta, distal splenic artery, superior and inferior mesenteric arteries, along with bilateral scattered areas of renal and splenic hypo perfusion. There was chronic total occlusion of the common iliac arteries, bilaterally. The thrombosed vessels showed mural thickening with progressive enhancement at delayed phases (Figure 2).

An urgent interventional radiology procedure was carried out utilizing the left brachial and radial arterial accesses, the angiogram revealed total aortoiliac occlusion, bilateral renal arteries and origin of the superior mesenteric arteries thrombosis (Figure 3).

PET scan showed mild symmetrical increased activity seen in both subclavian arteries of both upper arms and femoral arteries suggestive vasculitis.

Echocardiography revealed normal left ventricle size, ejection fraction 45 to $50 \%$, normal valves, and no overt intracardiac thrombus detected.

Rheumatological workup included: Antinuclear Antibodies (ANA), anti-double stranded DNA antibody (anti-dsDNA) (ELISA), anticardiolipin $\operatorname{Ig} G$, anticardiolipin IgA, anti- $\beta 2$ glycoprotein IgA, 


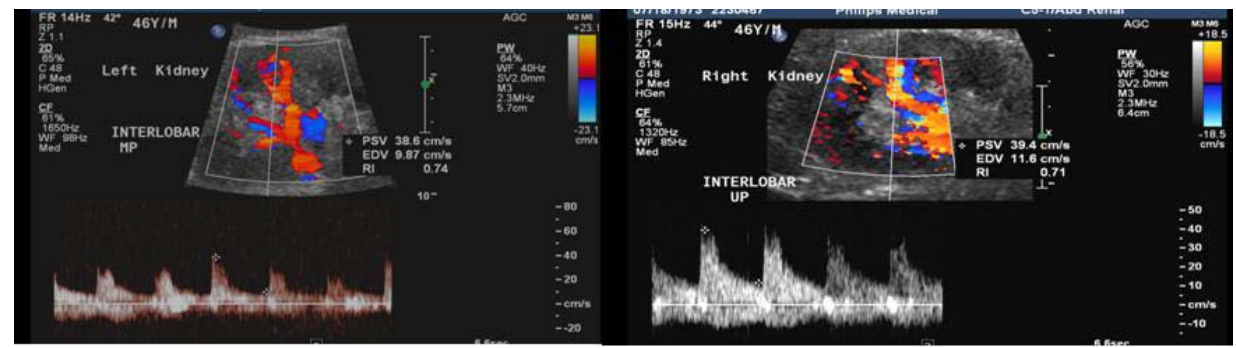

Figure 4: Selective DSA images of the renal artery after 12 hours of catheter directed thrombolysis shows patency of the renal arteries bilaterally with resumption of the flow to the renal artery bilaterally. Doppler ultrasound of the left and right kidney reveals resumption of the flow and improves peak velocity and resistive idices.

anti- $\beta 2$ glycoprotein IgG, anti- $\beta 2$ glycoprotein IgM, anti-Glomerular Basement Membrane antibody (anti-GBM), anti-neutrophil cytoplasmic antibodies (ANCAs), and perinuclear anti-neutrophil cytoplasmic antibodies (P-ANCA), which were all within normal range.

Hematological workup, such as gene testing for JAK 2 mutation and prothrombin mutation, Factor V Leiden was not detectable, Paroxysmal Nocturnal Hemoglobinuria (PNH) work-up was negative.

Antiphospholipid antibody work-up was repeated and came back negative again.

\section{Differential Diagnosis}

Important potential diagnoses of a patient presenting with bilateral renal artery thrombosis include: cardiac emboli secondary to atrial fibrillation or endocarditis, atheroemboli, large and medium vessel vasculitis, lupus vasculitis, sickle cell disease, fibromuscular dysplasia of the renal arteries, dissecting aneurysms of the aorta, and antiphospholipid antibody syndrome.

In this patient, there no previous past medical history of sickle cell disease, angioplasty, angiography, or cardiovascular surgery. No evidence of atrial fibrillation via ECG after reviewing all ECGs. Echo showed no vegetation and his blood cultures were negative. Initial unenhanced CT of his abdomen and pelvis showed no stones or hydronephrosis. Renal doppler US and CECT of his abdomen and pelvis confirmed the presence of aortic thrombosis extending to both renal arteries. Further hematological work-up needed in such a young patient included lupus antibodies, JAK-2 mutaion, antiphosolipid antibodies, Factor V Leiden, and prothrombin mutation, which were all unremarkable in our patient. Besides that, large vessels' vasculitis was part of the differential, so that PET scan was helpful in this case to confirm the diagnosis.

\section{Treatment}

Catheter-directed thrombolysis was done by intervention radiology through radial and brachial artery for bilateral renal artery thrombosis (Figure 4). Anticoagulation with intravenous heparin, then warfarin $3 \mathrm{mg}$ once daily with an International Normalized Ratio (INR) target of 2-3 indefinitely. Corticosteroids, specifically prednisolone, was started with a dose of $25 \mathrm{mg}$ per day, tapered down weekly by $5 \mathrm{mg}$ to reach a dose of $5 \mathrm{mg}$ daily. Methotrexate was initiated as well, with a dose of $10 \mathrm{mg}$ once per week.

\section{Outcome and Follow-Up}

Aortogram, the day after thrombolytic therapy, showed resumption of the flow to the renal artery bilaterally, and follow-up renal Doppler ultrasound confirmed no thrombus in renal arteries (Figure 4). The patient initially received 3 sessions of hemodialysis in the first few days of admission; however, after thrombolysis, he started to produce good urine output (around 3 liters per day).

After 4 months follow-up, his creatinine level was $113 \mathrm{u} \mathrm{mol} / \mathrm{L}$ (reached $1075 \mathrm{um} / \mathrm{L}$ upon admission), and he was passing good amount of urine with no overt new organ involvement.

\section{Discussion}

Takayasu Arteritis (TA) is a chronic granulomatous panarteritis, predominantly affecting females, but males are also affected [6]. The American Rheumatological Society considers three of the following six criteria necessary for a definite diagnosis of Takayasu's disease: age of onset $<40$, claudication of the limbs, decreased pulse of the brachial artery, difference in systolic blood pressure $>10 \mathrm{mmHg}$ between the two arms, murmurs of the subclavian arteries or the aorta, and abnormal arteriography [1]. Our patient exhibited three of the six criteria, with unknown age of onset and late diagnosis.

The clinical manifestations of TA are varied, and can be asymptomatic for several years, or can present as nonspecific systemic symptoms [2]. In this case, diagnosis of TA was delayed due to the nonspecific nature of the symptoms upon presentation with the absence of physical symptoms.

The course of the disease can be divided into an early active inflammatory phase, and a late chronic phase. The active phase involves fever, malaise, night sweats, loss of appetite, weight loss, headaches, dizziness, arthralgia, skin rashes, and more rarely acute vascular events. The late chronic phase is the result of arterial stenosis and/or occlusion, which primarily involves the aorta and its main branches, and the coronary and pulmonary arteries [3].

Among patients with TA, the prevalence of renal artery involvement was more common in Chinese patients compared to those in North America, Northern Europe, and Africa with renal artery stenosis and occlusion the most common abnormalities $[3,4]$. The angiographic classification of TA includes six findings: I: branches of aortic arch, IIa: ascending aorta, aortic arch and its branches, IIb: ascending aorta, aortic arch and its branches, and thoracic descending aorta, III: thoracic descending aorta, abdominal aorta, and/or renal arteries, IV: abdominal aorta and/or renal arteries, V: combination of 
Types IIb and IV [5,6]. Our patient was classified as TA, type IV, in which renal angiogram revealed bilateral renal arteries and origin of the superior mesenteric arteries with acute thrombosis.

Immunosuppressants such as prednisolone and methotrexate can lead to significant improvement in TA patients; however, around $50 \%$ of patients of TA progress to a stage that requires one or more surgical procedures, such as bypass grafts, angioplasty, or stent placement. In our case, thrombosis of the abdominal aorta and renal artery were the main cause of renal artery occlusion, such that the interventional radiology team was involved and successful catheterdirected thrombolysis was conducted.

TA is associated with favorable long-term renal and non renal outcomes [7]. The early initiation of therapy may result in reversible vessel pathology. This case report signifies the importance of early disease diagnosis and treatment which may result in significant renal improvement after sustained ischemia.

\section{Learning Points/Take Home Messages; 3-5 Bullet Points}

- Bilateral renal artery thrombosis is a rare cause of anuria and worsening kidney function, which needs to be diagnosed and treated when other sinister causes like obstructive uropathy are excluded.

- In addition to ischemia and stenosis, Takayasu vasculitis rarely presents with thrombosis formation, leading to significant organ damage that needs to be treated very early.
- Catheter-directed thrombolytic therapy for renal artery thrombosis results in rapid improvement in kidney function and prevents permanent severe renal damage and the need for renal replacement therapy.

\section{References}

1. Bloch DA, Michel BA, Hunder GG, McShane DJ, Arend WP, Calabrese LH, et al. The American College of Rheumatology 1990 criteria for the classification of vasculitis. Patients and methods. Arthritis Rheum. 1990; 33: 1068-1073.

2. Johnston SL, Lock RJ, Gompels MM. Takayasu arteritis: a review. J Clin Pathol. 2002; 55: 481-486.

3. Zhe Chen, Jing Li, Yunjiao Yang, Hongchao Li, Jiuliang Zho, Fei Sun, et al. The renal artery is involved in Chinese Takayasu's arteritis patients. et al. Kidney International. 2018; 93: 245-251.

4. Gudbrandsson B, Molberg O, Garen T, et al. Prevalence, incidence and disease characteristics of Takayasu Arteritis differ by ethnic background; data from a large, population based cohort resident in Southern Norway. Arthritis Care Res (Hoboken). 2017; 69: 278-285

5. Kerr GS, Hallahan CW, Giordano J, et al. Takayasu arteritis. Ann Intern Med. 1994; 120: 919-929.

6. Moriwaki R, Noda M, Yajima M, Sharma BK, Numano F. Clinical manifestations of Takayasu arteritis in India and Japan - new classification of angiographic findings. Angiology. 1997; 48: 369-379.

7. Baldwin C, Mohammad AJ, Cousins C, Carette S, Pagnoux C, Jayne D. Long-term outcomes of patients with Takayasu arteritis and renal artery involvement: a cohort study. Rheumatol Adv Pract. 2018; 2: rky026. 